\title{
The effect of loss-of-function allele (CYP2C19*3) with Clopidogrel efficacy in coronary heart disease patients
}

\author{
Ike Dhiah Rochmawati, Nur Hidayat, David Pomantow \\ Department of Clinical and Community Pharmacy, Faculty of Pharmacy, University of Surabaya, Indonesia
}

\section{Keywords \\ Antiplatelet \\ Clopidogrel \\ Coronary heart disease \\ CYP2C19*3 polymorphisms}

\section{Correspondence}

Ike Dhiah Rochmawati

Department of Clinical and Community Pharmacy

Faculty of Pharmacy

University of Surabaya

Jalan Raya Kalirungkut

Surabaya 60293

Indonesia

ike.dhiah@staff.ubaya.ac.id

\begin{abstract}
Introduction: Clopidogrel is the most widely prescribed antiplatelet for patients with coronary heart disease (CHD) who cannot take aspirin. Despite its effectiveness, Clopidogrel has several side effects caused by its metabolite. Clopidogrel resistance has been identified in some patients, and patient factors such as genetic polymorphisms in CYP2C19 may play a role in this resistance. The researchers wanted to look at $\mathrm{CYP} 2 \mathrm{C} 19 * 3$ polymorphisms and platelet aggregation in CHD patients who were taking clopidogrel. Methods: This research used a cross-sectional design. The research enrolled CHD patients at a local hospital's cardiology unit with certain inclusion and exclusion requirements. In the clinical laboratory, CYP2C19*3 polymorphisms was investigated using polymerase chain reaction (PCR), and platelet aggregation will be measured using light transmission aggregometry (LTA). Results: This research enlisted the participation of 53 patients. The majority of the patients $(68 \%)$ were men, with the highest age group being 60-69 years old. The most common comorbid disorder was hypertension. The result of $\mathrm{CYP} 2 \mathrm{C} 19 * 3$ polymorphisms as follows: GA (75\%), AA (21\%), and GG (4\%). Hypo-aggregation (89\%) and normalaggregation $(89 \%)$ are seen in the majority of patients $(11 \%)$. The authors were unable to locate the patient who had hyper-aggregation. Conclusion: According to descriptive research, CYP2C19*3 polymorphisms caused hypo-aggregation in more patients than normal aggregation in this study.
\end{abstract}

\section{Introduction}

The biggest health issues in the world nowadays are cardiovascular disease (CVD). Disorders in cardiovascular include conditions such as Coronary Heart Disease (CHD), Heart Failure, and Stroke. Cardiovascular disease is part of the NonCommunicable Diseases (NCD) and has surpassed cancer as the leading cause of death worldwide. Using data from the World Health Organization (WHO) in 2015 , it was said that $31 \%$ of 56.5 million deaths in the world were caused by cardiovascular disease. Some of them are CHD and stroke with $42.3 \%$ and $38.3 \%$, respectively. Deaths from heart disease occur in several countries, ranging from low-income to high-income countries. Indonesia is one of the developing countries with a high cardiovascular disease mortality rate (WHO, 2012).

In general, coronary heart disease can be divided into two, namely acute coronary syndrome and chronic coronary syndrome. The prevalence of $\mathrm{CHD}$ in Indonesia is still quite high, as evidenced by data submitted by the Basic Health Research by the Ministry of Health in 2014 showing the number of CHD patients in East Java based on a doctor's diagnosis by $0.5 \%$, while based on symptoms and a doctor's diagnosis by 1.3\% (Departemen Kesehatan, 2014). 
The accumulation of atherosclerotic plaque in the endothelial walls of coronary arteries causes CHD. Plaque builds up on artery walls, reducing the blood flow that carries nutrients and oxygen. Ischemic symptoms are reported by CHD patients due to an imbalance in oxygen supply and demand. The goal of CHD therapy is to keep the plaque in the patient's coronary arteries stable by inhibiting the progression of atherosclerotic plaque and preventing plaque that is formed from rupture (Montalescot, 2013).

One of the treatments for CHD patients is antiplatelet therapy. In CHD patients, antiplatelet therapy helps to avoid or minimize platelet aggregation and/or plaque formation. Reduced platelet aggregation and/or plaque formation lowers the risk of thrombus in blood vessels, thus lowers the risk of ischemia, which can further lead to ischemic cardiovascular events. Clopidogrel is an antiplatelet agent that is given as a treatment for coronary artery disease in addition to Aspirin. The CAPRIE study (Clopidogrel vs Aspirin in Patients at Risk of Ischemic Events) shows slightly better effectiveness on Clopidogrel compared to Aspirin in preventing cardiovascular events in patients with previous myocardial infarction, stroke, or peripheral arterial disease.

Clopidogrel's pharmacodynamic effects are dependent on factors that affect its metabolism, which is one of its drawbacks (Aradi, 2014). Clopidogrel is a prodrug that must be oxidized by the cytochrome P450 system in the liver to produce active metabolites. Clopidogrel's antiplatelet efficacy is determined by pharmacodynamic factors linked to drug-metabolising enzymes, such as cytochrome $\mathrm{P} 450$ classes (for example, CYP1A2, CYP2B6, CYP2C8, CYP2C9, CYP2C19, CYP3A4, and CYP3A5) and paraoxonase 1 (PON1). About $4-30 \%$ of patients treated with Clopidogrel show a low antiplatelet response or do not show an antiplatelet response. Clopidogrel's efficacy is influenced by a number of factors, including the patient's age, BMI (Body Mass Index), kidney disease, and genetic factors. From the genetic aspect, there are various CYP2C19 polymorphism profiles related to the metabolic process of the drug, where CYP2C19 is responsible for the metabolic activation of Clopidogrel, which is a prodrug to be transformed into active metabolites and loss-of-function alleles CYP2C19, which is directly related to the recurrence of cardiovascular disorders in patients receiving Clopidogrel. Clopidogrel resistance, or non-responsive Clopidogrel, is the term for this phenomenon. These patients, according to prior reports, have a higher risk of ischemic cardiovascular events.

CYP2C19*3 and CYP2C19*2 polymorphisms have the effect of reducing the effectiveness of Clopidogrel (lossof-alleles) active metabolites in the systemic and anti- aggregation ability. Judging from the genetic polymorphism present in CYP2C19, patients treated with Clopidogrel can show a varied therapeutic response (Yin, 2011).

It is well known that Clopidogrel is an inactive prodrug that requires oxidation by the hepatic cytochrome P450 system to produce active metabolites. Due to mutations in the gene for the CYP enzyme, this can affect the effectiveness of Clopidogrel. Of these genes, the main focus is CYP2C19. Loss-of-function alleles, such as CYP2C19*2 and CYP2C19*3, are responsible for decreased activation of Clopidogrel and increased risk of recurrent heart disease in CHD patients.

The prevalence of CYP2C19 loss-of-function polymorphisms worldwide is $24 \%$ of the non-Hispanic white population, $18 \%$ of Mexicans, $33 \%$ of African Americans, and $50 \%$ of Asians. This population is a homozygous carrier that has a poorly metabolised CYP2C19, thereby reducing the antiplatelet effect of Clopidogrel (Kitzmiller, 2011). The prevalence of CYP2C19 loss-of-function polymorphisms in Asia is $24.2 \%$ of the Japanese population, $14.8 \%$ of the Korean population, $8.9 \%$ of the Han Chinese, $9 \%$ of the Malay population and $13 \%$ of the Indian population. Specifically, in Singapore, there are $10 \%$ of ChineseSingaporean subjects and $9 \%$ of Malay-Singaporean subjects, and only $1 \%$ of Indian-Singaporean subjects (Chan, 2012).

The CYP2C19*3 is a loss-of-function allele, which is responsible for decreasing the activation of Clopidogrel. Clopidogrel concentrations in plasma are lower as a result of reduced clopidogrel activation, resulting in hyper platelet aggregation. The risk of persistent ischemia increases when hyper platelet aggregation occurs. Based on this background, and not many studies look at the genetic influence on the Clopidogrel metabolism process, the authors intend to conduct research by looking at the genetic influence on patients using Clopidogrel. The purpose of this study was to look at the effect of one of the alleles suspected of causing a decrease in the function of Clopidogrel (loss of function allele CYP2C19*3 polymorphisms). The effect of pharmacodynamic effects of Clopidogrel seen from platelet aggregation was measured using Light Aggregometry.

\section{Methods}

A cross-sectional design with descriptive analysis methods was used in this study. The aim of this study was to look at the relationship between CYP2C19*3 polymorphisms and platelet aggregation in $\mathrm{CHD}$ patients who were taking Clopidogrel. 


\section{Population of the study}

The participants in this study were patients with coronary artery disease who received outpatient treatment at a local hospital. The sample used is drawn from a population that meets the following inclusion and exclusion criteria:

\section{Inclusion criteria:}

1. Patients who are willing to join the study

2. Patients using generic clopidogrel therapy

3. Patients who have high adherence to treatment

4. Patients taking clopidogrel therapy for one month

\section{Exclusion Criteria:}

1. Patients undergoing chemotherapy

2. Patients who have liver problems

Before beginning the study, all participants signed a written informed consent form. The local hospital ethics committees have given their approval to the research.

\section{Genotyping}

The CYP2C19*3 polymorphism profile was analysed by the Polymerase Chain Reaction (PCR) method. The sample used was a patient's blood sample that DNA isolation stages had previously been carried out. PCR is used for DNA amplification using specific carrier primers. The primers used for CYP2C19*3 DNA amplification are primary pairs 5'TATTATTATCTGTTAACTAATATGA-3' and 5'ACTTCAGGGCTTGGTCAATA-3'. PCR was carried out in the following stages: initial denaturation at a temperature of $94^{\circ} \mathrm{C}$ for 2 minutes. After being denatured, it continues with 35 cycles consisting of:

a. Denaturation at $94^{\circ} \mathrm{C}$ for 45 seconds

b. Annealing at $53^{\circ} \mathrm{C}$ for 40 seconds

C. Polymerisation at $72^{\circ} \mathrm{C}$ for 30 seconds

d. Final extension at $72{ }^{\circ} \mathrm{C}$ for 5 minutes

After 35 cycles, the DNA amplicon is obtained. Followed by the digestion process, the CYP2C19*3 DNA amplicon was digested with the BamHI restriction enzyme at $37^{\circ} \mathrm{C}$ and a Bovine Serum Albumin (BSA) buffer. Deactivation of the enzyme at $65^{\circ} \mathrm{C}$ for 20 minutes. The next step was to obtain RFLP (Restriction Fragment Length Polymorphism) products. The obtained RFLP products are then separated in agarose gel solution (electrophoresis). The next step was the visualisation of electrophoresis results followed by staining in a solution of ethidium bromide $(5 \mathrm{mg} / \mathrm{mL})$ for ten minutes. Then the destaining step was carried out by immersing it in a solution of water for five-ten minutes.
After observing the migration of DNA in the UV lamp transilluminator. Then agarose gel which has been electrophoresed, and DNA migration was observed in UV transilluminator and then photographed.

\section{Platelet function test}

In this study, the profile of platelet aggregation was seen through the Turbidimetry test conducted at the Prodia Laboratory. The sample used in this test is platelet-rich plasma (PRP) from CHD patients using clopidogrel therapy. Platelet aggregation was measured in PRP at $37^{\circ} \mathrm{C}$ by light transmittance aggregometry using an aggregometer (Model 700, Chrono-Log Corp., USA). The PRP was pre-warmed to $37^{\circ} \mathrm{C}$ for five minutes before the addition of the agonists, i.e. $5 \mu \mathrm{M}$ adenosine diphosphate (ADP), 0.5 $\mathrm{mM}$ arachidonic acid (AA) and $2 \mu \mathrm{g} / \mathrm{ml}$ collagen (all from Chrono-Log Corp., USA). The aggregation response was monitored for at least five minutes, and the extent of aggregation was expressed as the percentage (\%) aggregation calculated using Aggrolink software (Chrono-Log Corp., USA)

\section{Results}

This research looked at patients with Coronary Heart Disease (CHD) who were taking Clopidogrel as their only antiplatelet medication. The study was carried out at the District General Hospital's Cardiology Center. A total of 53 samples were collected. Table I shows the patient characteristics in this report.

\section{Table I: Patient characteristics}

\begin{tabular}{lcc}
\hline Patient characteristics & $\mathbf{n}$ & $\mathbf{\%}$ \\
\hline Sex & & \\
- Male & 36 & $68 \%$ \\
- Female & 17 & $32 \%$ \\
\hline Age & & \\
- $40-49$ & 13 & $25 \%$ \\
- $50-59$ & 12 & $23 \%$ \\
- $60-69$ & 18 & $34 \%$ \\
- $70-74$ & 5 & $9 \%$ \\
- More than 75 & 5 & $9 \%$ \\
\hline Compelling indication & & \\
- Diabetes mellitus & 11 & \\
- Hypertension & 21 & \\
- Dyslipidemia & 5 & \\
- Heart Failure & 15 & \\
- Asthma & 1 & \\
- Chronic Kidney Disease & 2 & \\
- Without compelling indication & 8 & \\
\hline
\end{tabular}

Blood samples for platelet aggregation analysis must be processed within three hours of taking a patient's blood sample. This is achieved to eliminate platelet 
aggregation bias. Table II shows the outcomes of Light Transmission Aggregometry-assisted tests.

Table II: Profile of platelet aggregation with ADP as an inducer

\begin{tabular}{lcc}
\hline Type of platelet aggregation & $\mathbf{n}$ & $\mathbf{\%}$ \\
\hline Hypo-aggregation & 47 & $89 \%$ \\
Normal aggregation & 6 & $11 \%$ \\
Hyper-aggregation & 0 & 0 \\
\hline
\end{tabular}

CYP2C19*3 Polymorphisms were obtained by PCR and electrophoresis, and the results are shown in Table III.

Table III: Profile of CYP2C19*3 polymorphisms

\begin{tabular}{lcc}
\hline CYP2C19*3 polymorphisms & $\mathbf{n}$ & \% \\
\hline GA & 40 & $75 \%$ \\
GG & 2 & $4 \%$ \\
AA & 11 & $21 \%$ \\
\hline
\end{tabular}

The authors created a cross-tabulation between CYP2C19*3 polymorphisms and platelet aggregation. The result of cross-tabulation can be seen in Table IV.

Table IV: Cross-tabulation between CYP2C19*3 polymorphisms and platelet aggregation

\begin{tabular}{lccc}
\hline $\begin{array}{c}\text { CYP2C19*3 } \\
\text { Polymorphisms }\end{array}$ & $\begin{array}{c}\mathbf{n}(\%) \\
\text { Hypo- } \\
\text { aggregation }\end{array}$ & $\begin{array}{c}\text { Normal } \\
\text { aggregation }\end{array}$ & $\begin{array}{c}\text { Hyper- } \\
\text { aggregation }\end{array}$ \\
\hline GA & 35 & 5 & 0 \\
GG & 2 & 0 & 0 \\
AA & 10 & 1 & 0 \\
\hline
\end{tabular}

\section{Discussion}

The majority of the patients in the sample were male (68\%), with the majority of them being between the ages of 60 and 69 . There are a number of comorbid conditions, the most common of which is hypertension. Hypertension is a risk factor for coronary heart disease in principle. In the presence of hypertension, blood vessels may cause endothelial damage. Endothelial dysfunction, or damage to blood vessels, is the first stage of atherosclerotic plaque development. Patients with atherosclerotic plaque may experience complications such as coronary heart disease (CHD). According to the findings, almost all of the study participants had hypertension comorbidities, which supports the hypothesis that hypertension may be a cause of coronary heart disease.

Platelet aggregation measurements are measured using light transmission aggregometry (LTA). LTA is also known as optical turbidimetry or aggregometry. This LTA can be used to identify several disorders in platelet defects. Despite being used as a diagnostic tool, LTA can also be used to measure platelet function (Harrison, 2000; Michelson, 2004; Rand, 2003).

The decrease in optical density after stimulation of aggregation on platelet-rich plasma is used to measure platelet aggregation. This approach has many benefits, including the fact that it has been used in numerous studies for a long time, is predictable, and the instrument can be modified. There are some drawbacks to the benefits, such as the fact that it takes a long time, the sample preparation is very complex, and there is no simple standardisation (Favaloro, 2008; Lenk, 2013).

The agonists used in this test are ADP, collagen and epinephrine. Adding an agonist to this test aids to activate platelets. In this study, the only agonists observed were ADP. Clopidogrel drug inhibits platelet activation through inhibition of ADP binding to its receptors. ADP is removed from damage to blood vessels. ADP binding to $\mathrm{P} 2 \mathrm{Y} 12$ receptors causes changes in platelet shape and induction of platelet aggregation through internal calcium movement. ADP binding to $\mathrm{P} 2 \mathrm{Y} 12$ receptors plays a role in platelet aggregation response (Remijin, 2002; Koltai, 2017).

When the number of patients is divided by the degree of platelet aggregation, it can be shown that the number of patients with hypo-aggregation is much higher than the number of patients with normal platelet aggregation. The drug's pharmacokinetic and pharmacodynamic effects vary from patient to patient. Pharmacogenomic variation, which is genetic variation between people, is one explanation for the different responses. The enzymes involved in many stages of metabolism, such as the phase 1 reaction or the phase 2 reaction in drug metabolism, have been studied in pharmacogenomic studies. Cytochrome P450 (CYP450) is an enzyme that is involved in a variety of metabolic processes (Cacabelos, 2012).

Since the CYP2C group is responsible for about $20 \%$ of CYP450 substrate metabolism, genetic variation in one of the CYP2C9 classes induces different metabolism in certain CYP450 substrates (Speed, 2009; Liau, 2014; Wei, 2015; Brown, 2018).

According to the distribution of CYP2C19*3 polymorphisms in the study set, the findings of this study matches with Iddrisi and the authors (2018). The 
CYP2C19*3 polymorphism discovered was: $76.67 \%$ GA allele and $18.33 \%$ AA allele, according to the report. Due to its high prevalence relative to other enzymes, the CYP2C19 polymorphism is one of the most studied in Asian populations (Adithan, 2003; Johnson, 2011; Lyon, 2012; Strom, 2012).

CYP2C19*3 is a loss-of-function allele that causes clopidogrel activation to be reduced. Clopidogrel concentrations in plasma are lower as a result of reduced clopidogrel activation, increasing the risk of platelet hyper-aggregation if plaque on the walls of blood vessels ruptures. As platelets hyper aggregate, the chances of a thrombus forming increase. The presence of a thrombus will result in a blockage of the coronary arteries, and a blockage of the coronary arteries is the result of ischemia. This ischemia is a manifestation of coronary artery disease.

In this study, chi-square analysis calculations cannot be performed to see the relationship between CHD patients who have CYP2C19*3 polymorphisms with platelet aggregation because there is a value of 0 . According to theory, a loss-of-function CYP2C19*3 polymorphism could cause an increase in platelet aggregation. Clopidogrel metabolism into active metabolites is impaired due to CYP2C19*3 polymorphisms. Clopidogrel's active metabolites are essential for inhibitors of platelet activation to bind to P2Y12 receptors through ADP inhibition. In this study, CHD patients with CYP2C19*3 polymorphisms had mostly hypo-aggregation of platelets, which may be due to the presence of more than one CYP2C19 polymorphism in CHD patients.

It is also important to test clopidogrel drug concentration in CHD patients to determine the activity levels of Clopidogrel in the body. In different races, the CYP2C19*3 polymorphism is one of the strong determinants of decreased Clopidogrel active metabolites. Clopidogrel resistance is also influenced by the CYP2C19*3 polymorphism (Tresukusol, 2014). Based on Man and Chan's previous studies, the existence of CYP2C19*3 polymorphisms has an effect on Clopidogrel metabolic processes in Asian patients undergoing $\mathrm{PCl}$ (Percutaneous Coronary Intervention). Clopidogrel metabolism effects result in a decline in active Clopidogrel metabolites, which may affect Clopidogrel's antiplatelet activity.

Polymorphisms in the CYP2C19*2 and CYP2C19*3 genes have been related to HPPR (High PostTreatment Reactivity) in another study of patients with the acute coronary syndrome in Asian populations (Kim, 2009). Polymorphisms in the CYP2C19 gene are linked to high residual platelet reactivity, which raises the risk of major cardiovascular events (Yamamoto, 2011).
The development of thrombus in the coronary arteries is influenced by platelet reactivity. When a platelet is stimulated, the coagulation pathway opens, causing thrombin to be released. The presence of thrombin causes fibrin to activate, causing the thrombus to mature.

CYP2C19*3 polymorphism also has an effect on cardiovascular events. A study conducted by Jeong and the authors (2011) in patients with acute myocardial infarction in East Asia showed that the presence of CYP2C19*3 polymorphism was associated with an increase in cardiovascular events. Another study was conducted by Zhu (201)6 in patients undergoing Carotid Artery Stenting in Asia also showed a significant effect of CYP2C19*3 alleles on patient prognosis. Patients with CYP $2 \mathrm{C} 19 * 2$ and $* 3$ alleles have a higher ischemic event than those without.

\section{Limitations of the study}

Since we did not calculate plasma concentrations of Clopidogrel's active metabolite, we cannot provide clear proof of Clopidogrel's decreased antiplatelet efficacy in patients with at least one CYP2C19*3 variant allele.

\section{References}

Adithan, C., Gerard, N., Vasu, S., Rosemary, J., Shashindran, C.H., Krishnamoorthy, R. (2003). Allele and genotype frequency of CYP2C19 in a Tamilian population. Journal of Clinical Pharmacology, 56, 331-33. https://doi.org/10.1046/j.1365-2125.2003.01883.x

Aradi, D., Storey, R.F., Kom_ocsi, A., Trenk, D., Gulba, D., Kiss, R.G., Husted, S., Bonello, L., Sibbing, D., Collet, J.P., Huber, K. (2014). Working group on thrombosis of the European Society of Cardiology. Expert position paper on the role of platelet function testing in patients undergoing percutaneous coronary intervention. European Heart Journal, 35, 209-215. https://doi.org/10.1093/eurheartj/eht375

Brown, S.A., \& Pereira, N. (2018). Pharmacogenomic Impact of CYP2C19 variation on clopidogrel therapy in precision cardiovascular medicine. Journal of personalised medicine, 8(1), 8. https://doi.org/10.3390/jpm8010008

CAPRIE Steering Committee. (1996). A randomised, blinded, trial of Clopidogrel versus Aspirin in patients at risk of ischaemic events (CAPRIE). Lancet, 348, 1329-1339. https://doi.org/10.1016/s0140-6736(96)09457-3

Chan, Mark, Y. (2012). Clopidogrel Pharmacogenetics of east, south and other Asian populations. European Heart Journal Supplements, Volume 14, A41-A42. https://doi.org/10.1093/eurheartj/sur035 
Cacabelos, R. (2012). The Metabolomic Paradigm of Pharmacogenomics in Complex Disorders. Metabolomics 2, e119. https://doi.org/10.4172/2153-0769.1000e119

Department Kesehatan. (2014). Riset Kesehatan Dasar.

Favaloro, E.J. (2017). Clinical utility of closure times using the platelet function analyser-100/200. American journal of hematology, 92(4), https://doi.org/10.1002/ajh.24620

Harrison, P. (2000). Progress in the assessment of platelet function. British Journal of Haematology, 111, 733-44. https://doi.org/10.1111/j.1365-2141.2000.02269.x

Jeong, Y., Tantry, U.S., Kim, I., Koh, J., Kwon, T.J., Park, Y., Hwang, S., Bliden, K.P., Kwak, C.H., Hwang, J., Gurbel, P.A. (2011). Effect of CYP2C19*2 and CYP2C19*3 loss-of-function alleles on platelet reactivity and adverse clinical events in East Asian acute myocardial infarction survivors treated with Clopidogrel and Aspirin. Circulation Cardiovascular Interventions, 4 585-594. https://doi.org/10.1161/CIRCINTERVENTIONS.111.962555

Johnson, J.A., Gong, L., Whirl-Carrillo, M., Gage, B.F., Scott, S.A., Stein C.M., Anderson, J.L., Kimmel, S.E., Lee, M.T.M., Pirmohamed, M., Wadelius, M., Klein, T.E., Altman, R.B. (2011). Clinical Pharmacogenetics Implementation Consortium Guidelines for CYP2C9 and VKORC1 genotypes and warfarin dosing. Clinical Pharmacology \& Therapeutics, 90, 625-29. https://doi.org/10.1038/clpt.2011.185

Kim, I.S., Choi, B.R., Jeong, Y.H., Kwaks, C.H., Kim, S. (2009). The CYP2C19*2 and CYP2C19*3 polymorphisms are associated with high post-treatment platelet reactivity in Asian patients with acute coronary syndrome. Journal of Thrombosis and Haemostasis, 7, 897-899. https://doi.org/10.1111/j.1538-7836.2009.03319.x

Kitzmiller, J.P., Groen, D.K., Phelps, M.A., Sadee, W. (2011). Pharmacgenomic testing: relevance in medical practice: why drugs work in some patients but not in others. Cleveland Clinic Journal of Medicine, 78, 243-257. https://doi.org/10.3949/ccjm.78a.10145

Koltai, K., Kesmarky, G., Feher, G., Tibold, A., \& Toth, K. (2017). Platelet Aggregometry Testing: Molecular Mechanisms, Techniques and Clinical Implications. International journal of molecular sciences, 18(8), 1803. https://doi.org/10.3390/ijms18081803

Lenk, E., Spannagl, M. (2014). Platelet Function TestingGuided Antiplatelet Therapy. Electronic Journal of International Federation of Clinical Chemistry and Laboratory Medicine, 24(3): 90-96

Liau,Y., Muliaty, D. (2014). The Pharmacogenetics of Cytochrome P450 2C19 - enzymes Effects on Clopidogrel and Proton Pump Inhibitors. The Indonesia Biomedical Journal, 6(1), 33-44

Lyon, E., Foster, J.G., Palomaki, G.E., Pratt, V.M., Reynolds, K., Sabato, M.F., Scott, S.A., Vitazka, P. (2012). Laboratory testing of CYP2D6 alleles in relation to tamoxifen therapy. Genetics in Medicine, 14, 990-1000. https://doi.org/10.1038/gim.2012.108
Michelson, A.D. (2004).Platelet function testing in cardiovascular diseases. Circulation, 110(19), e489-e493. https://doi.org/10.1161/01.CIR.0000147228.29325.F9

Montalescot, G., Sechtem, U., Achenbach, S., Andreotti, F., Arden, C., Budaj, A., Yildirir, A. (2013). 2013 ESC guidelines on the management of stable coronary artery disease addenda. European Heart Journal, 34(38), 2949-3003. https://doi.org/10.1093/eurheartj/eht296

Rand, M. L., Leung, R., \& Packham, M. A. (2003). Platelet function assays. Transfusion and apheresis science. Official journal of the World Apheresis Association: official journal of the European Society for Haemapheresis, 28(3), 307-317. https://doi.org/10.1016/S1473-0502(03)00050-8

Remijin, J.A., Wu, Y., Jeninga, E.H., ljsseldijk, M.J.W., Willigen, G.V., Groot, P.G., Sixma, J.J., Nurden, A.T., Nurden, P. (2002). Role of ADP Receptor P2Y(12) in platelet adhesion and thrombus formation in flowing blood. Arteriosclerosis, Thrombosis, and Vascular Biology, 22, 686-691. https://doi.org/10.1161/01.ATV.0000012805.49079.23

Speed, W.C., Kang, S.P., Tuck, D.P., Harris, L.N., Kidd, K.K. (2009). Global variation in CYP2C8-CYP2C9 functional haplotypes. The Pharmacogenomics Journal, 9, 283-290. https://doi.org/10.1038/tpj.2009.10

Strom, C.M., Goos, D., Crossley, B., Zhang, K., Buller-Burkle, A., Jarvis, M., Quan, F., Peng, M., Sun, W. (2012). Testing for variants in CYP2C19: population frequencies and testing experience in a clinical laboratory. Genetics in Medicine, 14, 95- 100. https://doi.org/10.1038/gim.0b013e3182329870

Wei, Y.Q., Wang, D.G., Yang, H., Cao, H. (2015). Cytochrome P450 CYP 2C19*2 associated with adverse 1-year cardiovascular events in patients with acute coronary syndrome. PLOS ONE, 10(7), e0132561. https://doi.org/10.1371/journal.pone.0132561

World Health Organisation, World Heart Federation, World Stroke Organization. (2012). Global Atlas on Cardiovascular disease prevention and control. 155. https://doi.org/NLM classification: WG 120

Yamamoto, K., Hokimoto, S., Chitose, T., Morita, K., Ono, T., Kaikita, K., et al. (2011). Impact of CYP2C19 polymorphism on residual platelet reactivity in patients with coronary heart disease during antiplatelet therapy. Journal of Cardiology, 57, 194-201. https://doi.org/10.1016/j.jjcc.2010.10.007

Yin, T., Miyata, T. Pharmacogenomics of clopidogrel: Evidence and perspectives. Thrombosis Research. 2011, 128(4),

307-16. https://doi.org/10.1016/j.thromres.2011.04.010

Zhu, W.Y., Zhao, T., Xiong, X., Li, J., Wang, L., Zhu, Y., et al. 2016. Association of CYP2C19 polymorphisms with the clinical efficacy of clopidogrel therapy in patients undergoing carotid artery stenting in Asia. Scientific reports. 6:25478. https://doi.org/10.1038/srep25478 